\title{
Portable detection of clenbuterol using a smartphone-based electrochemical biosensor with electric field-driven acceleration
}

\author{
Yanzhi Dou $^{\mathrm{ab}}$, Zhineng Jiang ${ }^{\mathrm{b}}$, Wangping Deng ${ }^{\mathrm{b}}$, Jing Su ${ }^{\mathrm{b}}$, Shixing Chen ${ }^{\mathrm{b}}$, Haiyun \\ Song $^{\mathrm{c}}$, Ali Aldalbahi, ${ }^{\mathrm{d}}$ Xiaolei Zuo ${ }^{\mathrm{b}}$, Shiping Song ${ }^{\mathrm{b}, *}$, Jiye Shi, ${ }^{\mathrm{e}}$ Chunhai Fan ${ }^{\mathrm{b}, *}$ \\ ${ }^{a}$ Key Laboratory for Organic Electronics \& Information Displays (KLOEID), Institute of \\ Advanced Materials (IAM), National Syngerstic Innovation Center for Advanced Materials \\ (SICAM), Nanjing University of Posts \&Telecommunications, 9 Wenyuan Road, Nanjing 210023, \\ China \\ ${ }^{b}$ Division of Physical Biology \& Bioimaging Center, Shanghai Synchrotron Radiation Facility, \\ Shanghai Institute of Applied Physics, Chinese Academy of Sciences, Shanghai 201800, China \\ ${ }^{c}$ Key Laboratory of Food Safety Research, Institute for Nutritional Sciences, Shanghai Institutes \\ for Biological Sciences, Chinese Academy of sciences, Shanghai 200031, China \\ ${ }^{d}$ Chemistry Department, King Saud University, Riyadh 11451, Saudi Arabia \\ ${ }^{e}$ Kellogg College, University of Oxford, Oxford, OX2 6PN, UK
}

\begin{abstract}
We have developed a fast, highly sensitive and low-cost biosensing system for the detection of clenbuterol (CLB), using a homemade mobile electrochemical device with an electric field-driven acceleration strategy. This system consists of an embedded circuit in smartphone for signal processing and a screen-printed carbon electrode (SPCE) modified with multi-walled carbon nanobubes (MWNTs) and goat anti mouse-immunoglobulin G (IgG) sensing layer (MWNTs-I-layer). CLB monoclonal antibody was assembled through its binding to the surface-confined antibody. Such modified electrodes were used for rapid and sensitive amperometric immunosensing detection of CLB. Horseradish peroxidase-coupled CLB (CLB-HRP) competed with free CLB in the samples to bind the monoclonal antibody. By using this mobile system, we could detect CLB ranging from $0.3 \mathrm{ng} \cdot \mathrm{mL}^{-1}$ to $100 \mathrm{ng} \cdot \mathrm{mL}^{-1}$ with the detection limit of $0.076 \mathrm{ng} \cdot \mathrm{mL}^{-1}$. The whole competitive-type detection process was finished within 6 min. We expect this device can meet the requirements for field detection of various food security-related species.
\end{abstract}

\section{1. introduction}

Electrochemical analysis provides a vast array of important quantitative methods for detecting analytes, and has been widely demonstrated in many vital fields, including clinical diagnosis, environmental monitoring, homeland security, and food 
analysis[1-12]. In recent years, electrochemical immunosensing assay (ECIA) has gained considerable interest as an electrochemical bioanalytical method[13-17]. Although useful in a variety of settings, these methods are generally limited to well-resourced laboratories run by skilled personnel. Simultaneously, due to the lack of necessary equipment and devices in much of the developing world, especially in remote and rural areas, it is still a major challenge to communicate the results of testing with others. Thus, simplified and inexpensive versatile biosensing devices could become broadly applicable tools in the hands of healthcare workers, clinicians, farmers, and military personnel who need accurate and quantitative results in field, especially in resource-limited settings. Recently, in order to enable analytical devices to be used and communicated in any setting, the concept of using smart mobile phone has been proposed due to their ubiquitous availability and convenient connection to information networks for improving mobile detection[18,19]. Therefore, some devices are now being explored for point-of care (POC) diagnostics and environmental monitoring, aimed to develop useful mobile detection equipment. Even these devices are trying to use mobile phones for many different fields[20-23], few of them are being developed for detecting and analyzing mobile food security. Especially, many of them only provide qualitative ("yes" or "no") or visual readout, which can be ambiguous and difficult to interpret. However, only quantitative measurements offer an accurate and reproducible means for disease diagnosis and food security monitoring.

To combine smartphone with biosensors to develop a mobile detection system with multi-performance, we have designed a universal mobile and portable electrochemical device, which has both rapid electrochemical biosensing capability and information processing and communication property. Fig.1 shows the smartphone-based mobile electrochemical device connected to a biosensor chip, electrochemical detection component and electrical circuit frame diagram of the electrochemical device in smartphone. The smartphone-based electrochemical biosensing device has several advantages. First, an electrochemical detector was integrated into a smartphone, and a humanistic application (app) for the sensor is provided. Second, the most common electrochemical methods are available, including various forms of amperometry and voltammetry. Third, the smartphone-based electrochemical biosensor can operate as a modem to convey the results of testing to a remote facility through any available communications network $(2 \mathrm{G}, 3 \mathrm{G}$, WiFi, bluetooth). Forth, it supports extended memory (for instanse, the largest 32G/64G MicroSD) for saving data. It is ready for use at any time in our daily life. Especially, the smartphone-based device can also support electric field-driven acceleration for rapid immunosensing analysis.

Rapid immunosensing is highly desired for detection of proteins and drug molecules in POC testing. In order to accelerate the immunoreaction at the solid-liquid interface of electrodes, several techniques have been explored, including magnetic stirring, low-power microwave radiation, the electric field-driven and electrophoresis-assisted immunoassay [24-31]. Among these techniques, the electric field-driven method used to control nucleic acid hybridization, is an excellent 
enrichment and acceleration strategy for the transport of low-abundant protein and drug molecules. Positive driving potential was applied to accelerate the transport of negatively charged antigens to the electrode surface, while a low negative driving potential was applied to accelerate the transport of positively charged antigens to the electrode surface.

Herein, we apply our device as a smart mobile electrochemical detector by combining screen-printed carbon electrode chip with the electric field-driven acceleration strategy (Fig.2A). The integrated system can perform extremely easy-to-use, rapid, and portable electrochemical measurements. To demonstrate the availability of this system for POC testing, we designed an electric field-driven acceleration method for detection of clenbuterol (4-amino-[t-butylaminomethyl]-3,5-dichlorobenzyl alcohol hydrochloride, CLB). CLB was found to improve growth rate, reduce fat deposition and increase protein accretion so that it has been illegally used in livestock raising for the economical benefits[32]. However, CLB can easily remain in animal tissues and result in clinical symptom of human such as temporary dizziness and palpitations[33]. Accidents of CLB poisoning were found in some areas, even though it has been a banned feed additive in food-producing animals in most countries[34]. Therefore, the continuous surveillance of CLB abuse becomes seriously necessary for public health and the development of food industry. In our designed experiments, horseradish peroxidase-coupled CLB molecule (CLB-HRP) competed with free CLB molecule in the samples to bind to the monoclonal antibody modified on screen-printed carbon electrode surface by electric field-driven acceleration method (Fig.2C). Greatly improved sensitivity and shortened detection time are achieved by enzyme amplification and electric forces, demonstrating that the easy-to-use device has great promise for rapid and sensitive detection in other POC testing applications.

\section{Experimental}

\subsection{Reagents and materials}

MWNTs (50 $\mathrm{nm}$ in diameter, 1-2 $\mu \mathrm{m}$ in length) were purchased from Shenzhen Nanotech Port Co. Ltd. (Shenzhen, China). Bovine serum albumin (BSA) and Tween-20 were acquired from Sigma. Goat anti-mouse IgG was from Solaribio S\&T Co., Ltd.; Anti-CLB monoclonal antibody was purchased from Abcam Co.Ltd. Standard CLB sample was from Sigma. TMB (3,3,'5,5' tetramethylbenzidine) substrate was purchased from Neogen (Lexington, KY) in the format of a ready-to-use reagent (K-blue custom substrate, $\mathrm{H}_{2} \mathrm{O}_{2}$ included). PEG2000 was from Sigma.

All solutions were prepared with ultrapure water $\left(18 \Omega \mathrm{M} \mathrm{cm}^{-1}\right.$ resistivity) from a Millipore Milli-Q water purification system. Electrochemical detection was performed on a universal mobile and portable electrochemical device.

\subsection{Design of the mobile electrochemical device}


We designed the mobile electrochemical device based on customized Android application (app) to provide step-by-step instructions to facilitate user operation. The device includes three-electrode potentiostat, microcontroller (digital signals generator, digital collection system and digital processor), formed from electrochemical detection module to perform electrochemical measurements. The circuit design was showed in supporting information (see Fig. s1). After insertion of the biochip into the universal USB connection and link up to the phone, the Android operating system identified USB insertion signal. Menu was displayed on the home screen allowing the user to select from a list of processes and to intercalate corresponding experimental parameters. The device opened accessory protocol with the Android application to drive on digital signal generator to receive commands from user inputs and requested the date from the three-electrode potentiostat. Then, it carries out the electrochemical test and obtains the voltage and current signals from the biochip, and sent the data packets to digital collection system. The date was disposed by reassembling the messages and routed back to the phone in real-time with digital processor. Data visualization and analyses (denoising) was performed by the app on the phone.

\subsection{Purification and oxidation of MWNTs}

$5 \mathrm{~g}$ of MWNTs were immersed in concentrated hydrochloric acid for 48 hours, then centrifuged and washed with $0.1 \mathrm{M} \mathrm{NaOH}$ three times, and with Mill-Q water more than 3 times until the $\mathrm{pH}$ got neutral. The mixture was then reacted in a mixture of concentrated $\mathrm{HNO}_{3}$ and $\mathrm{H}_{2} \mathrm{SO}_{4}\left(\mathrm{v} / \mathrm{v}, 1: 3\right.$ ) for 10 hours at $60^{\circ} \mathrm{C}$ oil bath, and then centrifuged at $4000 \mathrm{rpm}$ for 30 minutes. The precipitate was reacted in a mixture of concentrated $\mathrm{H}_{2} \mathrm{SO}_{4}$ and $\mathrm{H}_{2} \mathrm{O}_{2}(\mathrm{v} / \mathrm{v}, 4: 1)$ for 30 minutes at $70^{\circ} \mathrm{C}$ oil bath, in order to further cut MWNTs and simultaneously increase amount of $-\mathrm{COOH}$ and $-\mathrm{OH}$. The reaction solution centrifuged at $4000 \mathrm{rpm}$ for 20 minutes to remove the supernatant solution, extensively washed in Milli-Q water and finally dried under vacuum. MWNTs after this treatment had solubility or high suspension stability without apparently aggregation for a long time at room temperature.

\subsection{Preparation of MWNTs-goat anti-mouse IgG conjugate}

$500 \mu \mathrm{L}$ of $1 \mathrm{mg} \cdot \mathrm{mL}^{-1} \mathrm{MWNTs}$ and $250 \mu \mathrm{L}$ of $2 \mathrm{mg} \cdot \mathrm{mL}^{-1}$ goat anti-mouse IgG were mixed together, and the mixture was incubated for 16 hours with vibration of $300 \mathrm{rpm}$ at $18{ }^{\circ} \mathrm{C}$. Then the mixture was centrifuged at $10000 \mathrm{rpm}$ for 10 minutes and washed with $750 \mu \mathrm{L}$ of Mill-Q water twice. Gently pipette up and down to resuspend the deposition if needed. To block the bare space on the surface of MWNTs, we resuspended the mixture with $600 \mu \mathrm{L}$ of $20 \%$ PEG2000 and the solution was subsequently incubated at $25{ }^{\circ} \mathrm{C}$ with vibration at $550 \mathrm{rpm}$ for 1.5 hours. Then the mixture was centrifuged and washed three times. $500 \mu \mathrm{L}$ Milli-Q was added to resuspend the resulting deposition by gently pipetting, and then the solution was moved to a new cuvette to be ready for next stage. 
2.5 Electrode surface modification and assembling of monoclonal antibody

Carbon screen-printed electrode chip were washed by Milli-Q and dried with $\mathrm{N}_{2}$ airflow, and then they were put into a refrigerator at $4{ }^{\circ} \mathrm{C}$ for 30 minutes. After cleaning, $6 \mu \mathrm{L}$ of MWNT-I conjugate from last stage was added onto the working electrode surface of each electrode. After airing at room temperature for at least 2 hours, $4 \mu \mathrm{L}$ of CLB monoclonal antibody solution $\left(4 \mu \mathrm{g} \cdot \mathrm{mL}^{-1}\right)$ was added onto the MWNT-IgG layer covered working electrode surface. Then the chips were kept in a humid space for 1.5 hours at $37{ }^{\circ} \mathrm{C}$. After rinsed with Milli-Q water and dried with air blowing, the chips were kept into a refrigerator at $4{ }^{\circ} \mathrm{C}$ for a long time.

\subsection{Preparation of clenbuterol-HRP conjugates (CLB-HRP)}

The molar ratio of CLB and HRP during conjugation is 10:1, where CLB was excessive according to a reported concentration optimization by ESI-MS (Roda et al., 2000). The unconjugated CLB would be removed in the following dialysis. The clenbuterol solution ( $0.5 \mathrm{mg}$ dissolved in $0.5 \mathrm{~mL}$ Milli-Q water) was mixed with 15 $\mu \mathrm{L}$ of $1 \mathrm{M} \mathrm{HCl}$ and $20 \mu \mathrm{L}$ of $1 \mathrm{M} \mathrm{NaNO}_{2}$ and vibrated for 15 minutes at $4{ }^{\circ} \mathrm{C}$. The HRP solution (11.9 mg HRP dissolved in $1 \mathrm{~mL}$ dilution buffer) was mixed with $20 \mu \mathrm{L}$ of $1 \mathrm{M} \mathrm{NaOH}$ and cooled to $4{ }^{\circ} \mathrm{C}$. The mixed clenbuterol solution was slowly added to the HRP solution. The mixed solution was then centrifuged at $300 \mathrm{rpm}$ at $4{ }^{\circ} \mathrm{C}$ for 2 hours. The resulting solution was dialyzed against $0.1 \mathrm{M} \mathrm{NaCl}$ and $0.2 \mathrm{M} \mathrm{PB}$ buffer (pH 7.4) for 1 day at $4{ }^{\circ} \mathrm{C}$ with agitation. $1 \mathrm{~mL}$ of glycol was added to the purification. The prepared CLB-HRP conjugate was stored at $-20^{\circ} \mathrm{C}$.

\subsection{Elecrtochemical detection of clenbuterol}

In the competitive immunoassay, 1:500 diluted CLB-HRP was firstly mixed with the clenbuterol standard sample at $1: 1$ volume ratio. Then $50 \mu \mathrm{L}$ of the mixture was added onto the electrode surface, reacted at a driving potential of $-0.3 \mathrm{~V}$ for 6 minutes. Free CLB-HRP was washed off with washing buffer. Electrochemical measurements were performed in TMB substrate at room temperature using the homemade smart mobile electrochemical device. Amperometric current versus time was measured at $-200 \mathrm{mV}$ (versus $\mathrm{Ag} / \mathrm{AgCl}$ reference electrode on the electrode chip) for $80 \mathrm{~s}$. Each electrode consisted of a carbon working electrode in the centre surrounded by an auxiliary electrode and an $\mathrm{Ag} / \mathrm{AgCl}$ reference electrode (see Fig. 2B).

The mixture of sample solution and CLB-HRP was dropped onto the electrode, and the whole system was incubated for 6 minutes by electric field-driven acceleration to finish the competitive immunoassay (see Fig.2C). Then the unbound CLB-HRP was removed in a washing step. When the TMB substrate was added, the bound CLB-HRP would subsequently catalyze the reduction of the TMB on the electrode surface, leading to a significantly amplified electrochemical current signal. 


\section{Results and Discussion}

\subsection{The design of Fast Electrochemical Immunosensor}

In this work, the principle of the immunosensor was based on the competition reaction between free CLB in samples and CLB-HRP conjugates for the limited anti-clenbuterol antibodies on the immunosensing-electrode surface. CLB monoclonal antibodies were assembled onto the nano-bio layer containing multi-walled carbon nanobubes and goat-anti-mouse IgG (MWNTs-IgG) to form ready-to-use immunesensing electrodes. In the absence of free CLB, CLB-HRP was mostly bound to monoclonal antibodies, resulting in the maximum current. In the presence of free CLB, CLB-HRP was partly or not bound to monoclonal antibodies, resulting in the decreased or blank current. In order to reduce time for competition reaction, the recognition process of the antigens and antibodies was driven by applying a negative driving potential for accelerating free CLB and CLB-HRP conjugates to arrive at electrode surface. In any case, the current signal was inversely related to the CLB concentration in samples. In the presence of $1 \mathrm{ng} \cdot \mathrm{mL}^{-1} \mathrm{CLB}$, a significant decrease of the catalytic reductive current was observed, indicating less CLB-HRP were bound to electrode surface because of the competition with free CLB molecules. Current signal for $0 \mathrm{ng} \cdot \mathrm{mL}^{-1}$ was $\mathrm{I}_{0}$, while I was for unknown samples or standard solutions. The percentage of the current reduction $\left(I_{R}=100 \times\left(1-I / I_{0}\right)\right.$ was used for the CLB quantification.

\subsection{Optimal Condition for Electric Field-Driven Incubation}

Since the immune competition reaction was related to the transport rates of antigens and antigen-conjugate to immunosensor interfaces, the transport rates of charged drug molecules could be accelerated after an electric field was produced near the electrode interfaces. Basically, the transport rates depended on solution $\mathrm{pH}$ value and driving potential. Thus, both the solution $\mathrm{pH}$ and driving potential could be optimized by monitoring the $\mathrm{I}_{\mathrm{R}}$ upon the competition reaction step. Notably, various antigen molecules would be differently charged in a specific buffer because of their different isoelectric points. In PBS buffer of $\mathrm{pH} 7.4$, the CLB molecules are positively charged due to its isoelectric point at $\mathrm{pH}$ 9.6. As shown in Fig.3, with the increase of negative driving potential, $I_{R}$ values changed at different critical driving potentials. With an incubation time of 6 minutes, $I_{R}$ values increased and then decreased along with the increase of critical driving potentials. The quick increase of $I_{R}$ indicated that the formation of immunocomplex (CLB monoclonal antibody-CLE) was accelerated at the immunosensing interfaces during a limited time, resulting in a lower current I based on the catalytic oxidation of HRP substrate 3,3,'5,5' tetramethylbenzidine (TMB) on the surface of the electrode. At high driving potential, the decrease of $I_{R}$ was attributed to the influence of the electrostatic repulsion among concentrated antigen molecules, resulting in the relatively low immunoreaction rate for the binding between the antigens to the immobilized monoclonal antibody on the surface of 
electrode with the driven transport rate. The critical driving potentials for CLB and CLB-HRP were determined to be $-0.3 \mathrm{~V}$.

At the driving potential of $-0.3 \mathrm{~V}$, upon the competition reaction between free CLB and CLB-HRP conjugates of 6 minutes, the $I_{R}$ increased, and then decreased until reached relatively stable values in the $\mathrm{pH}$ range of 5.5-8.0 (Fig. 4), indicating that the CLB antigen became positively charged at the solution $\mathrm{pH}$ lower than 9.6. At $\mathrm{pH}$ of 7.0, $\mathrm{I}_{\mathrm{R}}$ reached the highest value. Thus, PBS buffer of $\mathrm{pH} 7.0$ was used for the electric field-driven immune competition reaction.

The time for the electric field-driven immune competition reaction process was a crucial factor for an immunosensor. As shown in Fig. 5, at the driving potential of $-0.3 \mathrm{~V}$, with competition reaction time increasing, the $\mathrm{I}_{R}$ value reached to a relatively higher value. At 6 minute, $I_{R}$ value obtained with electric field-driven acceleration method was $61.9 \%$, more than 1.5 times compared to $I_{R}$ of $36.2 \%$ in ECIA. In addition, without electric field-driven acceleration, the competition reaction took 30 minutes with $I_{R}$ of $50.5 \%$. It was indicated that incubation with electric field-driven acceleration greatly improved the immune-binding efficiency. Thus, in order to reduce competition reaction time, 6 minutes was chosen for electric field-driven incubation.

\subsection{Optimization of Detection Condition}

The proposed method was based on the direct measurement of electrochemical signals produced by immobilized CLB-HRP. When CLB-HRP conjugates were bound to anti-CLB antibodies, the i-t curve showed the direct electrochemical response resulted from the catalytic oxidation of TMB by HRP. The signals were relevant to the acidity of the detection solution. The amperometric currents of CLB-HRP-labeled antibody of the immunosensors occurred at pH 7.4. Thus, $\mathrm{pH}$ 7.4 PBS buffer was used for signal detection.

\subsection{Electrochemical detection of CLE with electric field-driven acceleration strategy}

To demonstrate the advantages of the electric field-driven acceleration strategy, we compared it with the normal incubation strategy at room temperature. All electrodes were functionalized by MWNT-IgG layer and anti-CLB monoclonal antibodies, and then were used for detecting CLB samples $\left(0,0.3,1,3,10,10 \mathrm{ng} \cdot \mathrm{mL}^{-1}\right.$; three electrodes for each duplicate samples). The results were shown in Fig.6. Current signals from the immunosensors with electric field-driven acceleration were enhanced largely. Also, their current reduction ( $I_{R}$ values) was higher than that without electric field-driven acceleration, because a low negative driving potential was applied to accelerate the transport of positively charged CLB molecules to the electrode surfaces. Therefore, the electric field-driven acceleration strategy could help to reduce immune competition reaction time, improving the detection sensitivity and the signal-to-noise

ratio. Results also showed that $0.3 \mathrm{ng} \cdot \mathrm{mL}^{-1} \mathrm{CLB}$ could be detected with immunoelectrodes by electric field-driven acceleration strategy.

Using the smart biosensor, we detected clenbuterol solution of concentrations 
ranged from 0 to $100 \mathrm{ng} \cdot \mathrm{mL}^{-1}$ under optimized conditions using the electric field-driven acceleration strategy. The results were shown in Fig. 7. The fitted regression equation was as follows: $\mathrm{y}=(-3.910-77.505) /\left[1+(\mathrm{x} / 4.5281)^{1.04}\right]+77.505$ (x: clenbuterol concentration; $\mathrm{y}: \mathrm{I}_{\mathrm{R}}$ ). The detection limit corresponding to the signals of 3SD for the $\mathrm{I}_{\mathrm{R}}$ of clenbuterol was $0.076 \mathrm{ng} \cdot \mathrm{mL}^{-1}$, demonstrating the high sensitivity of the immunosensor. Notably, the detection limit of the commercial on-site assay for clenbuterol (e.g., gold immunochromatographic assay) was only about 3 ng.mL ${ }^{-1}$. Our smart immunosensor was at least 1 order of magnitude higher sensitive than most of the fast-detection methods based on portable chips or test strips. The advantage was probably derived from the high efficiency of the antibody assembling and fast competition reaction. The MWNT-IgG layer on the electrodes accurately controlled the interval between antibody molecules and improved the efficiency of the assembling of proteins.

\subsection{Spesitivity of the portable immunosensor}

To challenge the specificity of our amperometric immunosensor against other $\beta$-agonists that may exist in samples, we chose one representative analogues as interferons: ractopamine. Different concentrations of the interferents were measured with our amperometric immunosensor. The cross reactivity was calculated from the $I_{R}$. As shown in supplementary information (see Fig.s2), $I_{R}$ value increased $10 \%$ in the presence of 0.3 ng. $\mathrm{mL}^{-1}$ ractopamine molecules, indicating ractopamine combined with the assembled monoclonal antibodies. Since the specificity of immunosensors is dependent on the affinity of anti-CLB antibody, it can be improved by choosing high-affinity antibodies.

\section{Conclusion}

In this work, we have developed a smartphone-based immunosensor based on electric field-driven acceleration strategy for rapid and high-sensitivity detection of CLB molecules. The device takes advantage of the rapid and portable electrochemical instruments and the information-processing smart phones. Under optimal conditions, the immunosensor was able to detect a minimum of $0.076 \mathrm{ng} \cdot \mathrm{mL}^{-1}$ CLB in 6 minutes. And the device might have the potential to work with swine urine samples. The immunosensor is much more efficient for the detection of CLB than any other laboratory methods such as ELISA and HPLC and portable paper/stick-based methods. ${ }^{[5,34]}$. Combining different functionalized electrodes, this device can meet the requirements for field detection of all food security-related species. Thus, it would be a powerful tool for food security. Importantly, due to its high performance and appropriate size, the mobile biosensing system offers a possibility as an accessible, point-of-care platform, especially in remote and rural areas, holding a great promise for other mobile-testing requirements such as mobile health. 


\section{Acknowledgments}

We thank the National Basic Research Program of China (973 program, 2012CB932600 and 2013CB932800), the National Science Foundation of China (21373260 and 31571014), and Distinguished Scientist Fellowship Program of King Saud University for the financial support.

\section{References}

[1] X.-Y. Yu, Z.-G. Liu, X.-J. Huang, Nanostructured metal oxides/hydroxides-based electrochemical sensor for monitoring environmental micropollutants, Trac Trends. Environ. Anal.Chem. 3-4 (2014) 28-35.

[2] L'. Švorc, M. Rievaj, D. Bustin Green electrochemical sensor for environmental monitoring of pesticides: Determination of atrazine in river waters using a boron-doped diamond electrode, Sensors Actuators B: Chemical. 181 (2013) 294-300.

[3] L. Rassaei. Nanoparticles in electrochemical sensors for environmental monitoring, TrAC Trends .Anal. Chem. 30 (2011) 1704-1715.

[4] J.G. Pacheco, Castro. M, Machado .S, Fátima Barroso. M, Nouws. H.P.A, et a.I, Molecularly imprinted electrochemical sensor for ochratoxin A detection in food samples, Sensors Actuators B: Chemical. 215 (2015) 107-112.

[5] G. Liu, H.D. Chen, H.Z. Peng, S.p.Song, A carbon nanotube-based high-sensitivity electrochemical immunosensor for rapid and portable detection of clenbuterol, Biosens. Bioelectron. 28 (2011) 308-313.

[6] B. Liu, B. Xiao, L. Cui, M. Wang, Molecularly imprinted electrochemical sensor for the highly selective and sensitive determination of melamine, Mater Sci Eng C Mater Biol Appl. 55 (2015) 457-461.

[7] B. Kavosi, R. Hallaj, H. Teymourian, A. Salimi, Au nanoparticles/PAMAM dendrimer functionalized wired ethyleneamine-viologen as highly efficient interface for ultra-sensitive $\alpha$-fetoprotein electrochemical immunosensor, Biosens. Bioelectron. 59 (2014) 389-396.

[8] B. Jeong, R. Akter, O.H. Han, C.K. Rhee, M.A. Rahman, Increased Electrocatalyzed Performance through Dendrimer-Encapsulated Gold Nanoparticles and Carbon Nanotube-Assisted Multiple Bienzymatic Labels: Highly Sensitive Electrochemical Immunosensor for Protein Detection, Anal. Chem. 85 (2013) 1784-1791.

[9] Y. He, S. Xie, X. Yang, R. Yuan, Y. Chai, Electrochemical Peptide Biosensor Based on in Situ Silver Deposition for Detection of Prostate Specific Antigen, ACS Appl. Mater. Interfaces. 7 (2015) 13360-13366.

[10] Y. Du, Composition and architecture-engineered Au-SnO2/GNs-SWCNTs nanocomposites as ultrasensitive and robust electrochemical sensor for antioxidant additives in foods, Sensors Actuators B: Chemical. 203 (2014) 926-934.

[11] M. Barreiros dos Santos, Detection of pathogenic Bacteria by Electrochemical Impedance Spectroscopy: Influence of the immobilization strategies on the sensor performance, Procedia Chemistry. 1 (2009) 1291-1294.

[12] L. Asturias-Arribas, M.A. Alonso-Lomillo, O. Dominguez-Renedo, M.J. Arcos-Martinez, Sensitive and selective cocaine electrochemical detection using disposable sensors, Anal Chim Acta. 834 (2014) 30-36.

[13] V. Urbanova, Nanocrystalline Iron Oxides, Composites, and Related Materials as a Platform for 
Electrochemical, Magnetic, and Chemical Biosensors, Chem. Mater. 26 (2014) 6653-6673.

[14] J. Shen, Y. Li, H. Gu, F. Xia, X. Zuo, Recent Development of Sandwich Assay Based on the Nanobiotechnologies for Proteins, Nucleic Acids, Small Molecules, and lons, Chem. Rev. 114 (2014) 7631-7677.

[15] V. Mani, B.V. Chikkaveeraiah, V. Patel, J.S. Gutkind, J.F. Rusling, Ultrasensitive Immunosensor for Cancer Biomarker Proteins Using Gold Nanoparticle Film Electrodes and Multienzyme-Particle Amplification, ACS Nano. 3 (2009) 585-594.

[16] J. Das, M.A. Aziz, H. A. Yang, Nanocatalyst-Based Assay for Proteins: DNA-Free Ultrasensitive Electrochemical Detection Using Catalytic Reduction of $p$-Nitrophenol by Gold-Nanoparticle Labels, J. Amer. Chem. Soc. 128 (2006) 16022-16023.

[17] B.V. Chikkaveeraiah, A.A. Bhirde, N.Y. Morgan, H.S. Eden, X. Chen, Electrochemical Immunosensors for Detection of Cancer Protein Biomarkers, ACS Nano. 6 (2012) 6546-6561.

[18] Q.S. Wei, R. Nagi, K. Sadeghi, S. Feng, Detection and Spatial Mapping of Mercury Contamination in Water Samples Using a Smart-Phone, ACS Nano. 8 (2014) 1121-1129.

[19] P.B. Lillehoj, M.-C. Huang, N. Truong, C.-M. Ho, Rapid electrochemical detection on a mobile phone, Lab on a Chip. 13 (2013) 2950-2955.

[20] J.X.H. Wong, F.S.F. Liu, H.-Z. Yu, Mobile App-Based Quantitative Scanometric Analysis, Anal. Chem. 86 (2014) 11966-11971.

[21] J. Ryu, Y. Kim, B.H. Hong, S. Cho, Fast Synthesis of High-Performance Graphene Films by Hydrogen-Free Rapid Thermal Chemical Vapor Deposition, ACS Nano. 8 (2014) 950-956.

[22] A. Nemiroski, D.C. Christodouleas, J.W. Hennek, G.M. Whitesides, Universal mobile electrochemical detector designed for use in resource-limited applications, PNAS. 111 (2014) 11984-11989.

[23] J.L. Delaney, C.F. Hogan, J. Tian, W. Shen, Electrogenerated Chemiluminescence Detection in Paper-Based Microfluidic Sensors, Anal. Chem. 83 (2011) 1300-1306.

[24] E. Seyrek, P.L. Dubin, G.R. Newkome, Effect of Electric Field on the Mobility of Carboxyl-Terminated Dendrimers, J. Phys. Chem. B. 10 (2004) 10168-10171.

[25] M.J.R. Previte, K. Aslan, S.N. Malyn, C.D. Geddes, Microwave Triggered Metal Enhanced Chemiluminescence: Quantitative Protein Determination, Anal. Chem. 78 (2006) 8020-8027.

[26] J. Nie, Y. Zhang, H. Wang, S. Wang, G. Shen, Superhydrophobic surface-based magnetic electrochemical immunoassay for detection of Schistosoma japonicum antibodies, Biosens. Bioelectron. 33 (2012) 23-28.

[27] V.N. Morozov, T.Y. Morozova, Electrophoresis-Assisted Active Immunoassay, Anal. Chem. 75 (2003) 6813-6819.

[28] V.N. Morozov, S. Groves, M.J. Turell, C. Bailey, Three Minutes-Long Electrophoretically Assisted Zeptomolar Microfluidic Immunoassay with Magnetic-Beads Detection, J. Amer. Chem. Soc. 129 (2007) 12628-12629.

[29] D. Lin, J. Wu, M. Wang, F. Yan, H. Ju, Triple Signal Amplification of Graphene Film, Polybead Carried Gold Nanoparticles as Tracing Tag and Silver Deposition for Ultrasensitive Electrochemical Immunosensing, Anal. Chem. 84 (2012) 3662-3668.

[30] G. Lai, F. Yan, J. Wu, C. Leng, H. Ju, Ultrasensitive Multiplexed Immunoassay with Electrochemical Stripping Analysis of Silver Nanoparticles Catalytically Deposited by Gold Nanoparticles and Enzymatic Reaction, Anal. Chem. 83 (2011) 2726-2732.

[31] D. Du, J. Wang, D. Lu, A. Dohnalkova, Y. Lin, Multiplexed Electrochemical Immunoassay of 
Phosphorylated Proteins Based on Enzyme-Functionalized Gold Nanorod Labels and Electric Field-Driven Acceleration, Anal. Chem. 83 (2011) 6580-6585.

[32] R.J. Murphy, L. Beliveau, K.L. Seburn, P.F. Gardiner, Clenbuterol has a greater influence on untrained than on previously trained skeletal muscle in rats, Eur J Appl Physiol Occup Physiol. 73 (1996) 304-310.

[33] J. Mbuna, T. Kaneta, T. Imasaka, Rapid determination of multidrug resistance-associated protein in cancer cells by capillary electrophoresis immunoassay, J Chromatogr A. 1218 (2011) 3923-3927.

[34] S.X. Li, J. Zhang, W.p. Deng, X.Z. Shen, S.P. Song, C.H. Fan, A Highly Sensitive Amperometric Immunosensor for Clenbuterol Detection in Livestock Urine, Electroanalysis. 25 (2013) 867-873. 


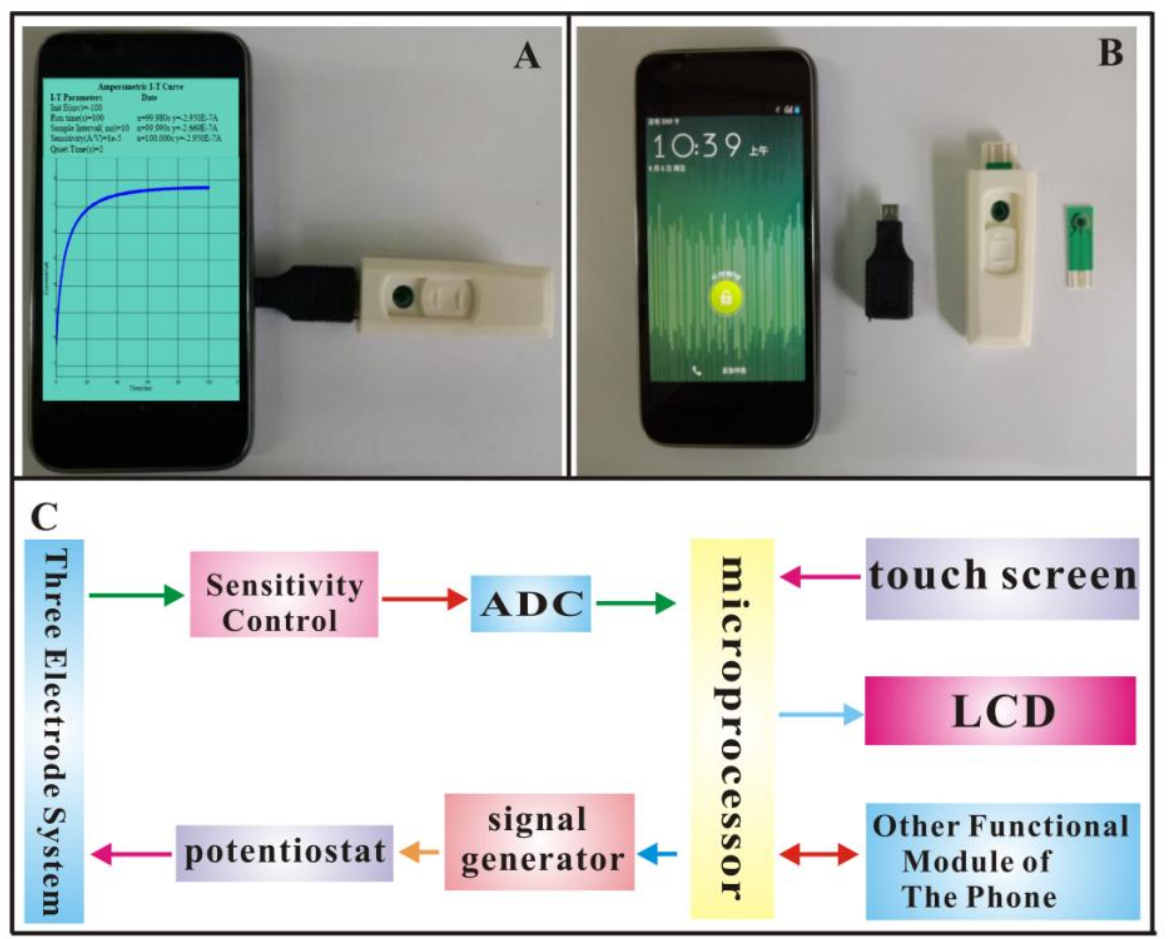

Fig. 1 (A) A photograph of the smartphone-based electrochemical biosensor. (B) A photograph of the smartphone, USB port, chipbox and screen-printed carbon electrode. (C) An electrical circuit frame diagram of the mobile electrochemical device, enables parameter input from the user to the mobile phone and data collection from three electrode system connected with the phone. (ADC: analog to-digital converter. LCD: liquid crystal display.)

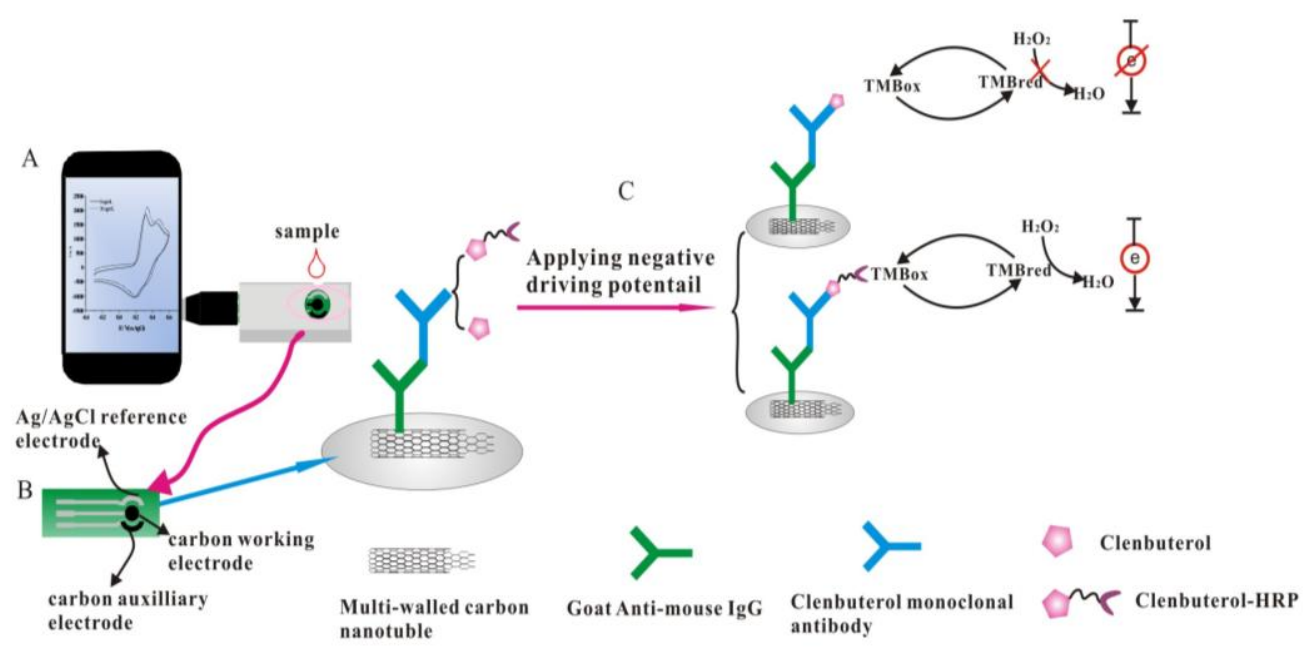

Fig. 2 Design of the electrochemical immunosensor for CLE detection with an electric field-driven incubation process; (A) homemade smartphone-based electrochemical device; (B) screen-printed electrode chip; (C) free clenbuterol competed with CLB-HRP to bind to the limited anti- clenbuterol antibody on the electrode surface. CLB-HRP combined with anti- clenbuterol antibody catalyzed the electrochemical reaction of TMB substrate solution to obtain a detective current signal. 


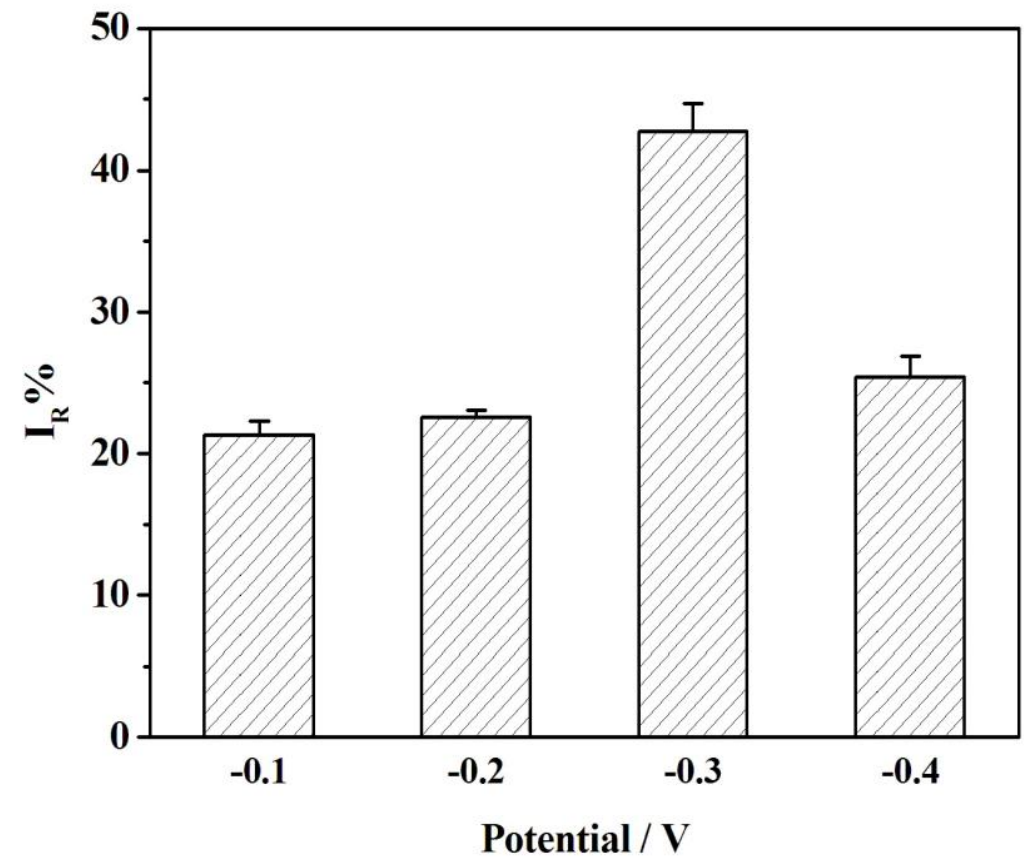

Fig. 3 Effect of driving potential for electric field-driven competition reaction (immunosensing responses after 6 minutes at room temperature). The reaction solution is $10 \mathrm{mM}$ containing 10 ng. $\mathrm{mL}^{-1}$ CLB molecules (2\% BSA in $10 \mathrm{mM} \mathrm{pH} 6.5 \mathrm{PBS}$ ).

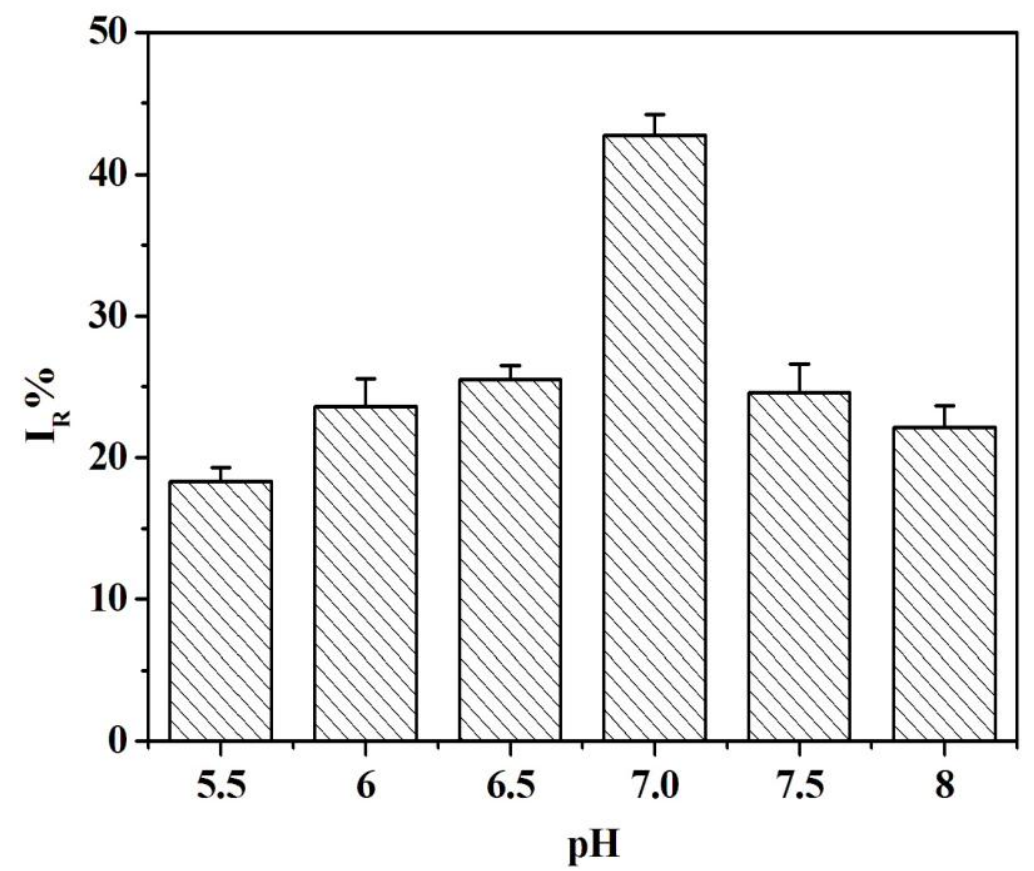

Fig. 4 Effect of competition reaction solution $\mathrm{pH}$ values at a driving potential of $-0.3 \mathrm{~V}$ for electric field-driven competition reaction (immunosensing responses against $10 \mathrm{mM}$ different $\mathrm{pH}$ values PBS after 6 minutes at room temperature). The reaction solution is $10 \mathrm{mM}$ PBS containing $2 \%$ 
BSA and 3 ng. $\mathrm{mL}^{-1}$ CLB molecules.

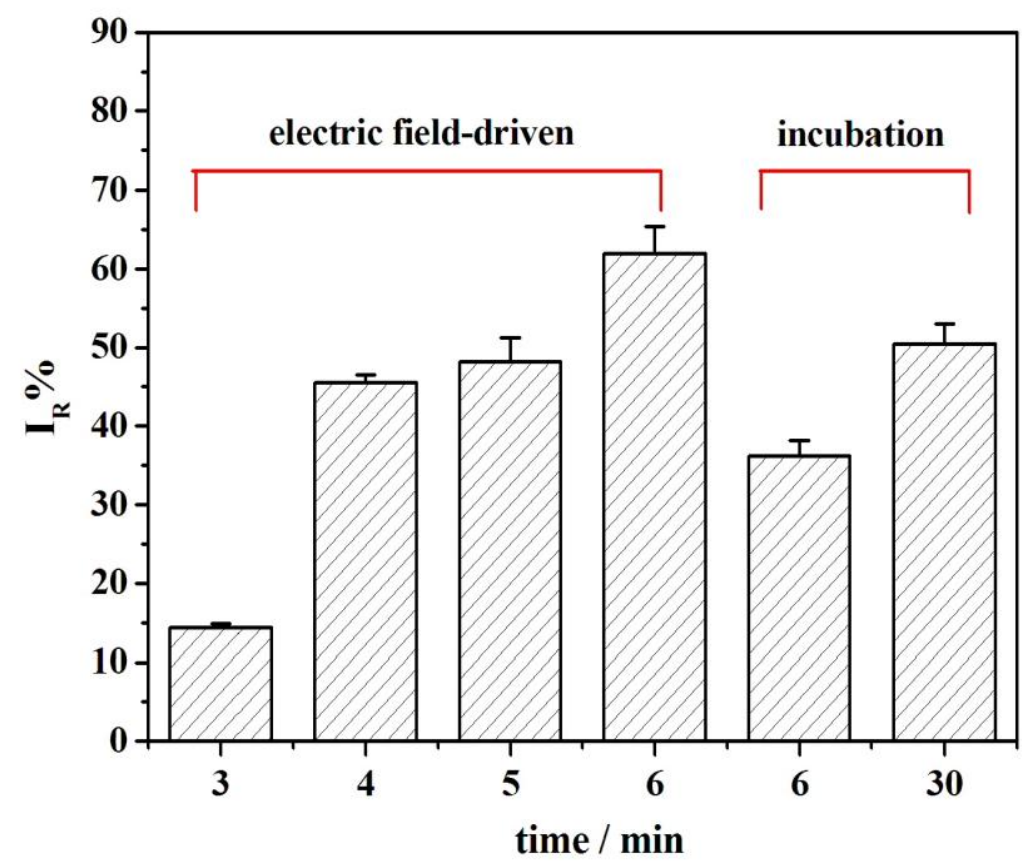

Fig. 5 Effects of electric field-driven competition reaction time at a driving potential of $-0.3 \mathrm{~V}$ at room temperature (in $10 \mathrm{mM} \mathrm{pH}$ 7.0 PBS reaction solution containing $2 \%$ BSA and $3 \mathrm{ng} \cdot \mathrm{mL}^{-1}$ CLB molecules).

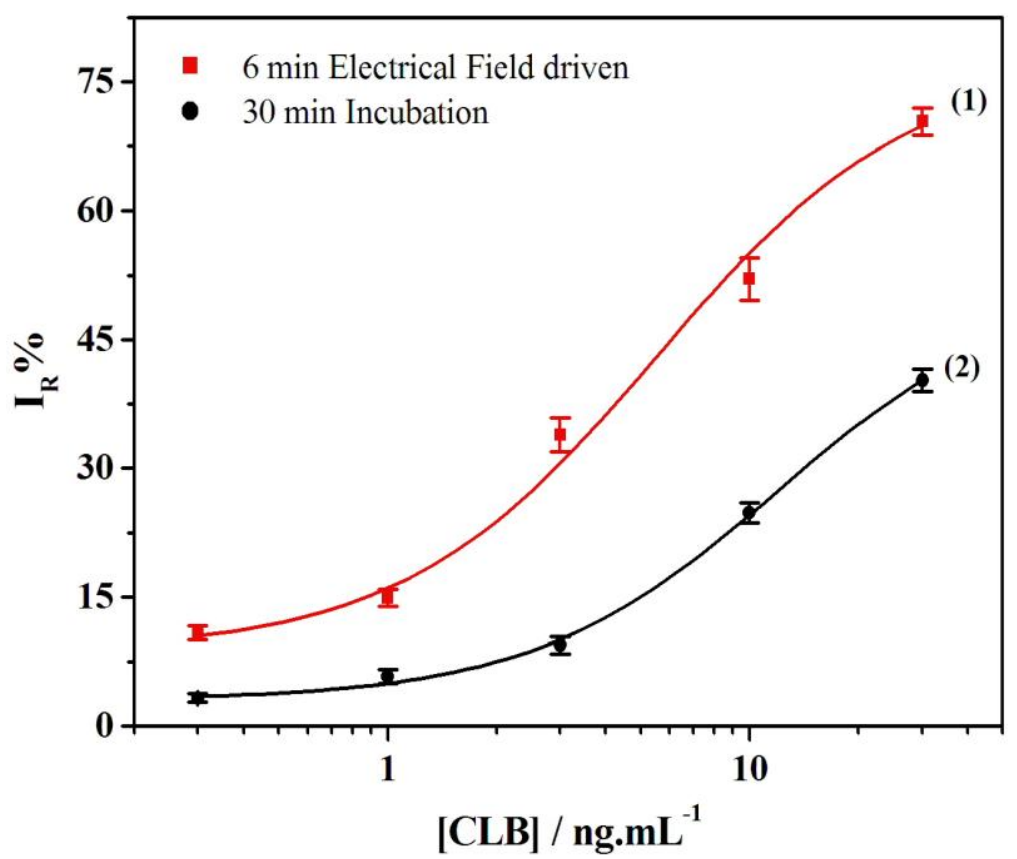

Fig. 6 Immunosensor with different competition reaction strategies were used to analysis five 
CLB samples. (1) 6 minutes electric field-driven at a driving potential of $-0.3 \mathrm{~V}$ in $10 \mathrm{mM} \mathrm{pH} 6.96$ PBS reaction solution containing $2 \%$ BSA. (2) 30 minutes incubation in $10 \mathrm{mM}$ pH 7.0 PBS reaction solution containing $2 \% \mathrm{BSA}$. The concentrations of CLB was $0.3,1,3,10,30 \mathrm{ng} \cdot \mathrm{mL}^{-1}$.
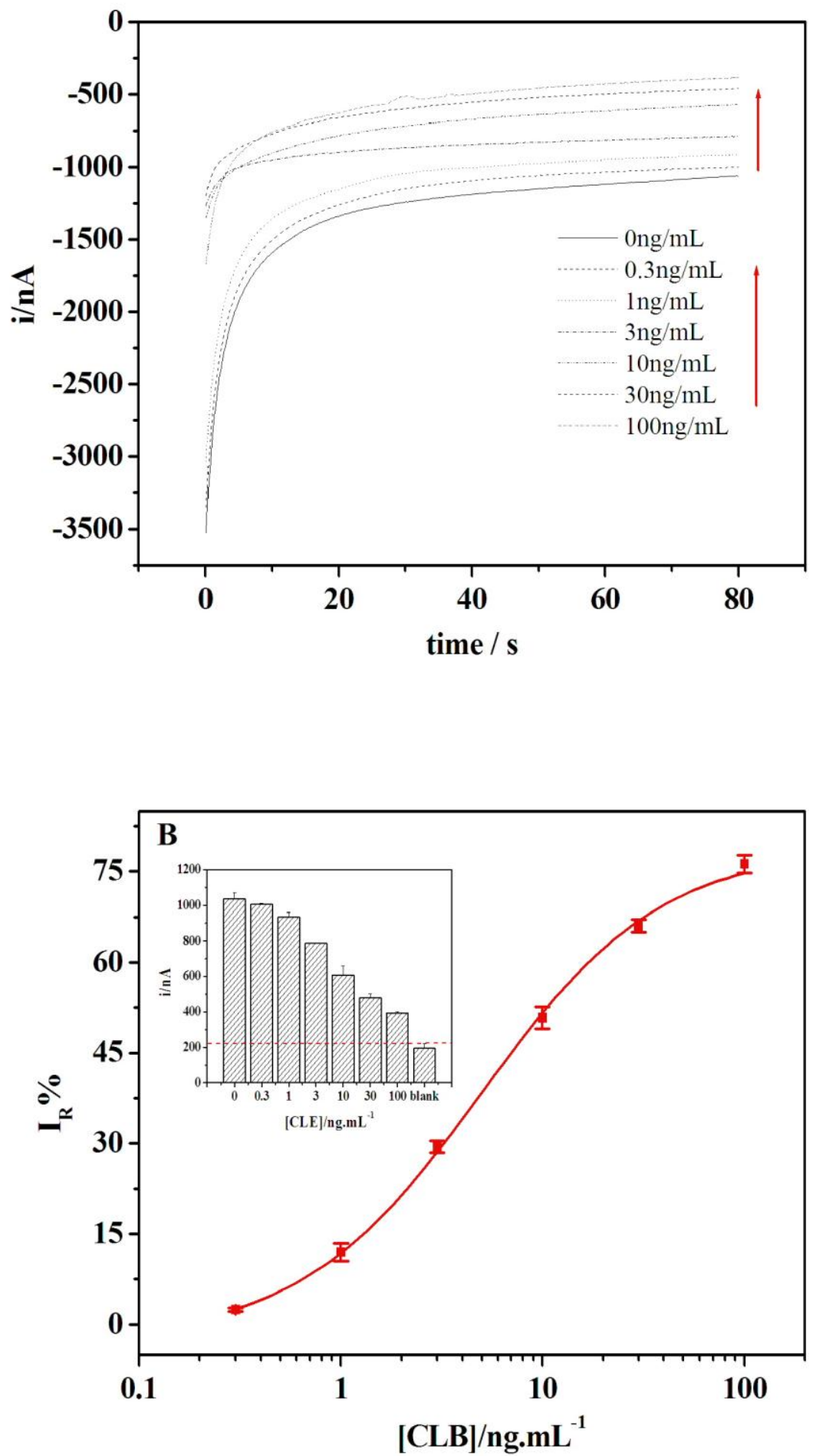

Fig. 7 (A) Amperometric responses results with electric field-driven compition reaction at $0.3 \mathrm{~V}$ for 6 minutes to increasing CLB concentrations $\left(0,0.3,1,3,10,30\right.$ and 100 ng.mL $\left.{ }^{-1}\right)$. (B)The resulted calibration curves. 